\title{
Universal finite-size scaling amplitudes of interfacial free energies in Monte Carlo simulations
}

\author{
Hyunggyu Park $\dagger$ and Marcel den Nijs \\ Department of Physics, FM-15, University of Washington, Seattle, Washington 98195, USA
}

Received 23 August 1988, in final form 3 February 1989

\begin{abstract}
The study of the universal finite-size scaling amplitudes of interfacial free energies in Monte Carlo simulations provides an accurate method of determining the critical temperature and the universality class of phase transitions. These universal amplitudes can replace the critical exponents to identify universality classes. We use Bennett's optimisation scheme for the calculation of the interfacial free energies in Monte Carlo simulations. Tests for the Ising and three-state Potts model confirm the effectiveness of this method.
\end{abstract}

Finite-size scaling (FSS) is an important tool in numerical investigation of critical phenomena (Nightingale 1976, 1982, Barber 1983). In particular it has been applied very successfully to two-dimensional models on semi-infinite lattices in the context of transfer matrix calculations. Recently the type of analysis that employs the universality of the finite-size scaling amplitudes of correlation lengths and interfacial free energies (Luck 1982, Cardy 1984) has improved the numerical accuracy of the transfer matrix method considerably (Nightingale and Blöte 1983, Blöte and den Nijs 1988). However, transfer matrix calculations are limited to relatively small strip widths $L$; the required computer memory increases exponentially with $L$, as $q^{L}$ with $q$ the number of states per site or per bond. Therefore the transfer matrix method is not an adequate tool to study systems with large $q$, nor systems that require a large lattice to exhibit the structure of their ordered phases properly, e.g. commensurate phases with a large unit cell or incommensurate phases (for a review, see den Nijs 1988). Monte Carlo (MC) simulations are more suited for these types of problems. In this paper we introduce universalamplitude finite-size scaling to MC simulations.

Consider a $L_{1} \times L_{2}$ lattice. We investigate the FSS behaviour of the interfacial free energies $\eta$ which are defined as the difference between the free energies per unit length for different boundary conditions. For example, in the Ising model the free energy of an interface (Bloch wall) is equal to the difference in free energy between a system with periodic and antiperiodic boundary conditions. The interfacial free energy is finite in the ordered phase, vanishes in the disordered phase, and scales at criticality as

$$
\eta\left(L_{1}, L_{2}\right) \simeq A(s) / L_{2}
$$

with $s=L_{2} / L_{1}$ the aspect ratio. This is an elementary consequence of the scaling postulate (Nightingale 1976, 1982, Privman and Fisher 1984). The amplitudes $A(s)$ are universal and vary continuously with the aspect ratio $s$.

The main purpose of this paper is to point out and illustrate that also in MC simulations it pays to focus on these universal amplitudes. To identify the universality

$\dagger$ Present address: Department of Physics, Carnegie-Mellon University, Pittsburgh, PA 15213, USA. 
class of a phase transition from the Fss behaviour of e.g. the specific heat (Landau 1976)

$$
C\left(L_{1}, L_{2}\right) \sim B(s) L_{2}^{2 y_{T}-D}
$$

with $D=2$ being the dimension of the system, one needs to fit two parameters: the amplitude $B(s)$ which is not universal, and the thermal critical exponent $y_{\mathrm{T}}$ which is universal (one needs to subtract a regular background term as well). Compare this with (1). The universal amplitudes require only a one-parameter fit. This becomes even more advantageous in MC simulations than in transfer matrix calculations. In the transfer matrix method free energies can be obtained numerically with machine accuracy, and therefore both types of fits work well. In MC simulations ensembleaveraged quantities like order parameters, specific heats, and interfacial free energies have a typical accuracy about $0.1 \%$, due to statistical fluctuations, for the relatively small system sizes we need consider.

Can these universal amplitudes replace the critical exponents as the parameters that identify the universality class of a phase transition? Is it possible to deduce the values of the critical exponents from the values of the universal amplitudes? Otherwise one still needs to perform a two-parameter fit of the first derivative of $\eta$ with respect to temperature, $\mathrm{d} \eta / \mathrm{d} T \cong A(s) L_{2}^{y_{T}{ }^{-1}}$ (or magnetic field), to determine the critical exponents. The relation between the universal amplitudes and the critical exponents is simple in the limit of semi-infinite strips, i.e. in transfer matrix calculations: $A(0)=$ $2 \pi x$ with $x$ the critical dimension of the operator conjugate to the interface imposed by the boundary condition. For example, for cyclic boundary conditions (antiperiodic boundary conditions in the Ising model) $x$ is equal to the magnetic critical exponent $x=x_{H}$ (Cardy 1984, Park and den Nijs 1988).

The connection between critical exponents and universal amplitudes becomes more complex on a finite-by-finite lattice (a torus), i.e. in MC simulations. Park and den Nijs (1988) list the possible boundary conditions for the $2 \mathrm{D} q$-state Potts model on a torus, and derive the exact values of $A(s)$ for all aspect ratios $s$. We use the so-called extended-scaling method (den Nijs 1979, 1983, 1984, Nienhuis 1987). The exact values of the critical exponents of most $2 \mathrm{D}$ phase transitions have been determined in recent years by extended scaling (for a review, see Nienhuis 1987) and by conformal invariance (Belavin et al 1984, Friedan et al 1984; for a review see Cardy 1987). For every 2D universality class where these methods can be applied, it will be possible to determine the exact values of the universal amplitudes as well.

We will not compare the accuracy of our test results with Monte Carlo Fss results that use (2), but rather with those of the more sophisticated Monte Carlo real-space renormalization (MCRG) method (Swendsen 1979, 1982). However it should be noted that real-space RG methods are unsuited to several types of problems to which we intend to use our method later, e.g. commensurate-incommensurate transitions (the domain wall periodicity varies continuously and therefore is incompatible with a specific fixed block-spin periodicity).

MC algorithms for the interfacial free energies have been developed by several authors (Bennett 1976, Mon and Jasnow 1984, 1985, Mon and Nightingale 1985). We use the optimisation scheme proposed by Bennett (1976), and employ gauge invariance to reduce the statistical errors in the MC results. Consider two systems with Hamiltonians $H_{0}$ and $H_{1}$. The free energy difference between the two systems, $\Delta F \equiv F_{1}-F_{0}$, can be written in terms of ensemble averages as

$$
\exp (\Delta F)=\left\langle W \exp \left(-H_{0}\right)\right\rangle_{H_{1}} /\left\langle W \exp \left(-H_{1}\right)\right\rangle_{H_{0}}
$$


where $W$ is arbitrary. Bennett (1976) showed that $W^{-1}=Z_{0} \exp \left(-H_{1}\right)+Z_{1} \exp \left(-H_{0}\right)$ is the optimal choice for the function $W$ which minimises the statistical errors under the Gaussian-noise assumption of ensemble-averaged quantities. $Z_{0}$ and $Z_{1}$ are the partition functions of the two systems. With this choice, (3) can be written as

$$
\left\langle f\left(H_{1}-H_{0}-\Delta F\right)\right\rangle_{H_{0}}=\left\langle f\left(H_{0}-H_{1}+\Delta F\right)\right\rangle_{H_{1}}
$$

where $f(x)=1 /\left(1+\mathrm{e}^{x}\right)$. This is an implicit equation for $\Delta F$. We run two MC simulations; one with Hamiltonian $H_{0}$ and one with $H_{1}$. During both simulations we store the probability distribution $P(\Delta H)$, with $\Delta H=H_{1}-H_{0}$. Afterwards $\Delta F$ follows easily by solving the equation

$$
\sum_{h} P_{0}(h) f(h-\Delta F)=\sum_{h} P_{1}(h) f(\Delta F-h) .
$$

This method works well when there is sufficient overlap between the two ensembles. In our case $H_{0}$ and $H_{1}$ differ only by boundary conditions.

Consider the ferromagnetic $q$-state Potts model with a spin $\sigma(n, m)$ on each site of a square lattice, and nearest-neighbour interactions $-K \delta_{\sigma, \sigma^{\prime}}$. In the vertical direction the boundary condition is periodic, $\sigma\left(n, m+L_{1}\right)=\sigma(n, m)$. In the horizontal direction the boundary condition is periodic, $\sigma\left(n+L_{2}, m\right)=\sigma(n, m)$ in the case of Hamiltonian $H_{0}$, while it is cyclic, $\sigma\left(n+L_{2}, m\right)=\sigma(n, m)+1$ in the case of $H_{1}$. The latter is equivalent to periodic boundary conditions but with modified interactions $-K \delta_{\sigma, \sigma^{\prime}+1}$ instead of $-K \delta_{\sigma, \sigma^{\prime}}$ at all bonds that cross a seam that runs across the lattice in the vertical direction (see figure 1). So $\Delta H$ is equal to $K \delta_{\sigma, \sigma^{\prime}}-K \delta_{\sigma, \sigma^{\prime}+1}$ summed over all bonds that cross the seam. There are many more types of possible boundary conditions, e.g. twisted ones as discussed in our paper (Park and den Nijs 1988). They are equally interesting, but here we restrict ourselves to cyclic boundary conditions.

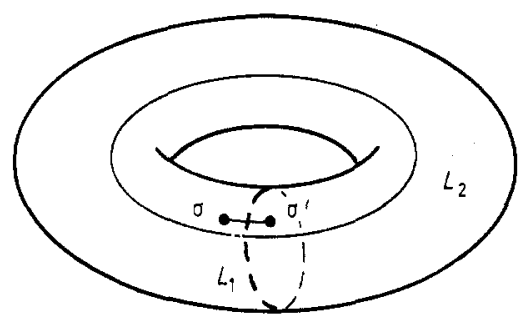

Figure 1. Cyclic boundary conditions for a Potts model on a $L_{1} \times L_{2}$ lattice. The vertical broken line represents the seam where the interactions are modified as $-K \delta_{\sigma, \sigma^{\prime}+1}$.

The cyclic boundary condition induces an interface in the low-temperature phase. The interfacial free energy is defined as the difference in free energy per unit length between the two systems

$$
\eta\left(L_{1}, L_{2}\right)=\Delta F / L_{1}=\left(1 / L_{1}\right) \ln \left(Z_{0} / Z_{1}\right) .
$$

Gauge invariance implies that the shape and the location of the seam is arbitrary (Park and den Nijs 1988). We consider only seams that form straight vertical lines, and denote the Hamiltonian $H_{1}$ with a seam at position $n=x$ by $H(x)$ (figure 1). We use the remaining gauge invariance (the position of the seam) to reduce the statistical error in $\Delta F$. We sum over all possible locations of the seams,

$$
\sum_{x=1}^{L_{2}}\left\langle f\left(H(x)-H_{0}-\Delta F\right)\right\rangle_{H_{0}}=\sum_{x=1}^{L_{2}}\left\langle f\left(H_{0}-H(x)+\Delta F\right)\right\rangle_{H(x)}
$$


The summation on the left-hand side can be moved inside the average, but the one on the right-hand side seems to involve $L_{2}$ different MC simulations. It is easy to show by gauge invariance that the seams of the Hamiltonians used in the ensemble and those inside the average can be separated as

$$
\left\langle f\left(H_{0}-H(x)+\Delta F\right)\right\rangle_{H(x)}=\left\langle f\left(H^{\prime}(x)-H_{0}+\Delta F\right)\right\rangle_{H\left(x_{0}\right)}
$$

if $x \neq x_{0}$. The seams in $H^{\prime}(x)$ and $H\left(x_{0}\right)$ are now at different positions. The prime in $H^{\prime}(x)$ indicates that at its seam the interactions are modified in the opposite direction, as $K \delta_{\sigma, \sigma^{\prime}-1}$ instead of $K \delta_{\sigma, \sigma^{\prime}+1}$. Equation (8) allows us to evaluate (7) from two MC simulations only; one with Hamiltonians $H_{0}$ and one with $H\left(x_{0}\right)$. This way of averaging over all seam positions enhances the accuracy of the MC results significantly, especially for large lattices.

First, we test our method to the $2 \mathrm{D}$ Ising model on a square lattice. In order to determine the critical temperature and the universal amplitude, we use the conventional extrapolation method used in transfer matrix calculations (Nightingale 1982, Nightingale and Blöte 1983, Blöte and den Nijs 1988). Interfacial free energies $\eta$ are calculated on the $4 \times 4$ and $6 \times 6$ lattice at temperatures $\exp (-K)=0.41$ and 0.42 . In the $\left(\exp (-K), \eta L_{2}\right)$ plane, we connect the points with the same lattice size by straight lines. The temperature $\exp (-K)$ and the amplitude $\eta L_{2}$ at the crossing point of these two straight lines are recorded. Next, interfacial free energies are calculated on the $6 \times 6$ and $8 \times 8$ lattice at the temperature of the previous crossing point and one of the two initial temperatures, 0.4 or 0.42 . We iterate the above procedure.

We consider lattice sizes $4 \times 4,6 \times 6,8 \times 8$, and $10 \times 10$. We use respectively $2,3,4$, and 5 independent $10^{6} \mathrm{MC}$ steps per spin (MCS) for the calculation of the interfacial free energy at a given temperature. The initial $10^{4} \mathrm{MCS}$ are discarded to reach equilibrium. Statistical errors in the values of the $\eta\left(L_{2}\right)$ are about $0.1-0.15 \%$. We established these error estimates by comparing results for independent MC runs.

The temperatures and the amplitudes of the three crossing points using the above iteration method are plotted in figure $2(a)$ and $(b)$. Extrapolations of these points to $1 / L_{2}=0$ using the expected type of asymptotic behaviour, as described below, yield that $\exp \left(-K_{\mathrm{c}}\right)=0.4142 \pm 0.0006$ and $A(s=1)=0.987 \pm 0.016$. The exact values of the critical temperature and the universal amplitude at aspect ratio $s=1$ are $\exp \left(-K_{\mathrm{c}}\right)=$ $\sqrt{2}-1$ and $A(1)=\ln \left(1+2^{3 / 4}\right)=0.9865$ (Park and den Nijs 1988). The Ising model is special, because it is exactly soluble on a finite-by-finite lattice (Kaufman 1949, Ferdinand and Fisher 1969). Therefore not only these asymptotic values, but also the exact values of $\eta\left(L_{1}, L_{2}\right)$ at all values of $L_{1}$ and $L_{2}$ are known. Our numerical results are in excellent agreement with these exact values.

In the limit $L_{2} \rightarrow \infty$, the crossing points asymptotically must approach the exact values of the critical temperature and the universal amplitude, $\exp \left(-K_{\mathrm{c}}\right)$ and $A(s)$, as

$$
\exp \left(K^{*}\right)-\exp \left(K_{\mathrm{c}}\right) \sim L_{2}^{-y_{\mathrm{T}}+y_{\mathrm{ir}}}
$$

and

$$
\eta^{*} L_{2}-A(s) \sim L_{2}^{y_{\text {ir }}}
$$

where $K^{*}$ and $\eta^{*}$ are the values of temperature and interfacial free energy at the crossing points. $y_{\mathrm{T}}$ is the thermal exponent and $y_{\mathrm{ir}}$ the leading correction-to-scaling exponent. Equations (9) and (10) follow from elementary scaling theory. It is assumed that the temperature scaling field does not vary with $N$. This is a consequence of self-duality at the fixed point, and also follows from conformal theory. 

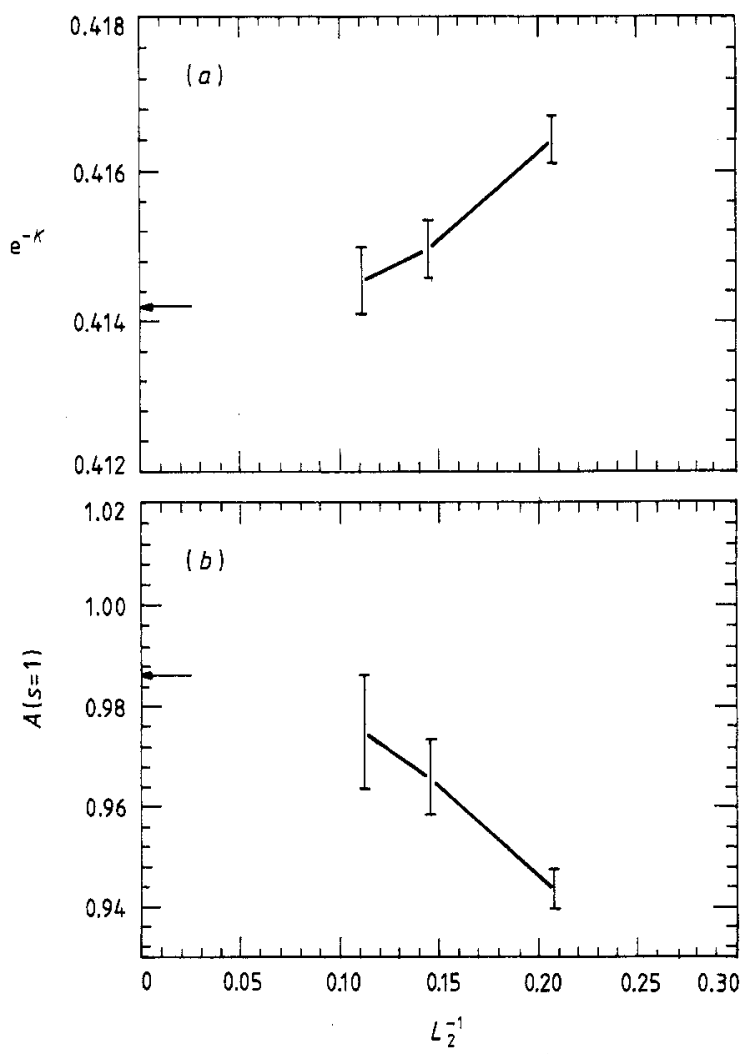

Figure 2. Convergence of the crossing points in the Ising model. The temperatures $(a)$ and the amplitudes (b) of the crossing points are plotted as functions of $1 / L_{2}$. Arrows indicate the exact values for the critical temperature $(a)$ and the universal amplitudes $(b)$. The full lines between the Monte Carlo data are guides to the eye.

It is useful to comment on how well (9) and (10) work in transfer matrix finite-size scaling calculations in semi-infinite strips for the Ising model and the three-state Potts model (den Nijs and Park, unpublished; see also e.g. Blöte and den Nijs 1988). In the Ising model it is known that the leading irrelevant exponent has the value $y_{\mathrm{ir}}=-2$. Indeed the corrections to scaling in the semi-infinite case follow (9) and (10) with this value of $y_{\text {ir }}$ very accurately already at small strip widths $N<10$. In the three-state Potts model the convergence is less rapid. If one fits the numerical transfer matrix data at successive crossing points to the form (9) and (10), and then extrapolate these approximants for the critical exponents $y_{\mathrm{T}}$ and $y_{\mathrm{ir}}$, one finds indeed the known exact values of the temperature and leading irrelevant critical exponents $y_{\mathrm{T}}=\frac{6}{5}$ and $y_{\mathrm{ir}}=-\frac{4}{5}$, each with an accuracy better than a few per cent. This confirms numerically that (9) and (10) are correct. However, in the three-state Potts model the asymptotic scaling region where only one irrelevant operator dominates has not been reached for strip widths $N \leqslant 10$. We expect the presence of a subdominant irrelevant operator with exponent $y_{\mathrm{ir}}=-2$ with a large amplitude. The Potts model can be mapped onto the BCSOS model (see e.g. Park and den Nijs 1988) and this surface roughening model is likely to have a large correction to scaling associated with the Gaussian operator $(\nabla \phi)^{4}$ similar to the restricted solid-on-solid model where this has previously been found to be the case numerically (den $\mathrm{Nijs} 1985 \mathrm{~b}$ ). Indeed a fit of the transfer matrix results 
to the version of (9) and (10) with two irrelevant operators, assuming the values $y_{\mathrm{ir}, 1}=-\frac{4}{5}$ and $y_{\mathrm{ir}, 2}=-2$, leads to stable amplitudes, with an amplitude of the $y_{\mathrm{ir}, 2}=-2$ term which is ten times larger than that of the $y_{\mathrm{ir}, 1}=-\frac{4}{5}$ term.

On the torus the corrections to scaling in the crossing point temperatures and universal amplitudes must be of the same order of magnitude as in semi-infinite strips, but the statistical error in the MC data prevents these types of very detailed extrapolations. In principle one could increase the length of the MC runs until the statistical error is small enough to make an unbiased extrapolation possible. Our MC runs are not long enough yet for that. We obtained the above mentioned results for the Ising model, $\exp \left(-K_{\mathrm{c}}\right)=0.4142 \pm 0.0006$ and $A(s=1)=0.987 \pm 0.016$, by extrapolating the MC results, using (9) and (10) with $y_{\mathrm{ir}}=-2.0$. In other words, this type of accuracy, with our statistical error, requires prior knowledge of the order of magnitude of the critical exponents. For example, a $1 / N$ extrapolation to the crossing point temperatures can be ruled out. In cases where the orders of magnitude of $y_{\mathrm{T}}$ and $y_{\mathrm{ir}}$ are completely unknown, one has several options. First, to determine the crossing points at finite $N$ with more accuracy by running more MC simulations at different temperatures. Next, study larger lattice sizes or to improve the statistics of the smaller sizes. Our results confirm that the asymptotic scaling region, dominated by the dominant irrelevant operators, is reached at the same lattice sizes as in semi-infinite lattices. This suggests that one should opt for improving the statistics at small lattice sizes. Notice that the system sizes that we consider, sizes customary in transfer matrix calculations, are quite small compared with what is customary in Monte Carlo simulations.

In many cases the critical temperature $\exp \left(-K_{c}\right)$ is already known, for example from duality. Then it is not necessary to determine crossing points. The universal amplitude can then be obtained by extrapolating $\eta\left(L_{2}\right)$ at $\exp \left(-K_{\mathrm{c}}\right)$. Figure 3 shows that this also works very well in our MC method. We show the exact curve and our numerical results. In figure 3 the only uncertainty is the statistical error in the values of $\eta\left(L_{2}\right)$. Now the lengths of the MC runs that we consider $\left(5 \times 10^{6} \mathrm{MCs}\right)$ are sufficient to make an unbiased extrapolation, using (10), feasible. We find $A(s)=0.987 \pm 0.003$, and the numerical value of $y_{\mathrm{ir}}$ is consistent with the exact value $y_{\mathrm{ir}}=-2$.

If the universality class is known, for example, from the symmetry of the system, then the critical temperature can be determined with high accuracy from the knowledge

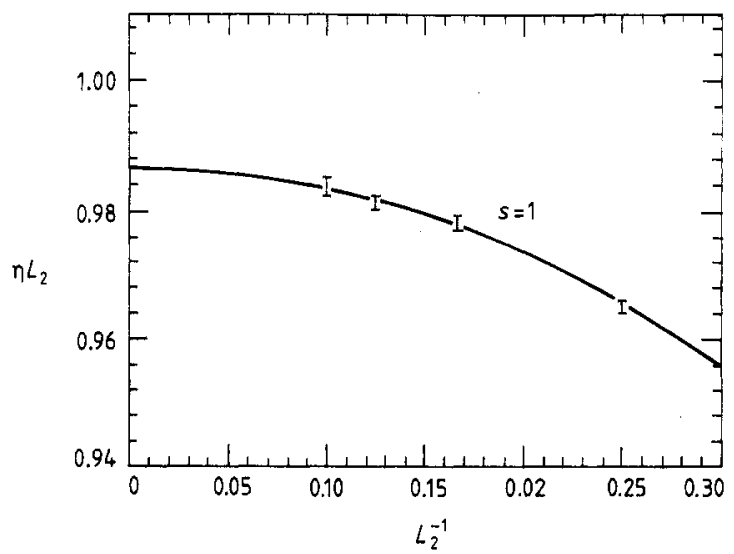

Figure 3. Universal amplitudes of the Ising model. Monte Carlo data at $L_{2}=4,6,8$ and 10 are consistent with the exact curve (the full curve) within error bars. 
of the value of the universal amplitude $A(s)$. We obtain $\exp \left(-K_{\mathrm{c}}\right)=0.4142 \pm 0.0001$; see figure 4 .

The $2 \mathrm{D}$ Ising model is special; the model is exactly soluble and the structure of the corrections to scaling is particularly simple (Blöte and den Nijs 1988). Therefore we also tested our method on the three-state Potts model. In figure 5, we plot the numerical values of $\eta L_{2}$ at the critical temperature as function of $1 / L_{2}$ for the same lattice sizes as before for aspect ratios $s=1,0.75$ and 0.5 . In this case we needed MC runs twice as long as in the Ising model to obtain the same accuracy for the interfacial free

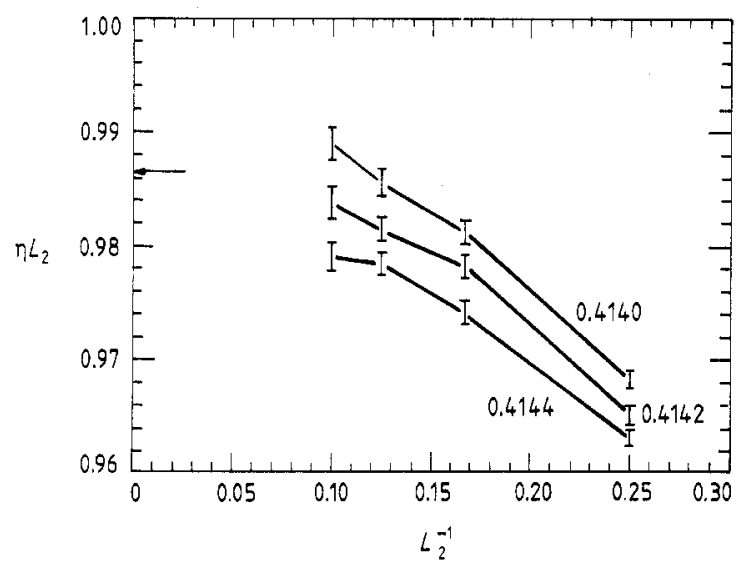

Figure 4. The critical temperature of the Ising model. Monte Carlo data for $L_{2}=4,6,8$ and 10 at temperatures $\exp (-K)=0.4140,0.4142$ and 0.4144 are shown with error bars. The full lines between the Monte Carlo data are guides to the eye. The line at the critical temperature must approach the exact value of the universal amplitude $A(s=1)=0.9865$ (indicated by the arrow) in the limit $L_{2} \rightarrow \infty$. The line at $\exp (-K)=0.4140(0.4144)$ approaches clearly above (below) the arrow.

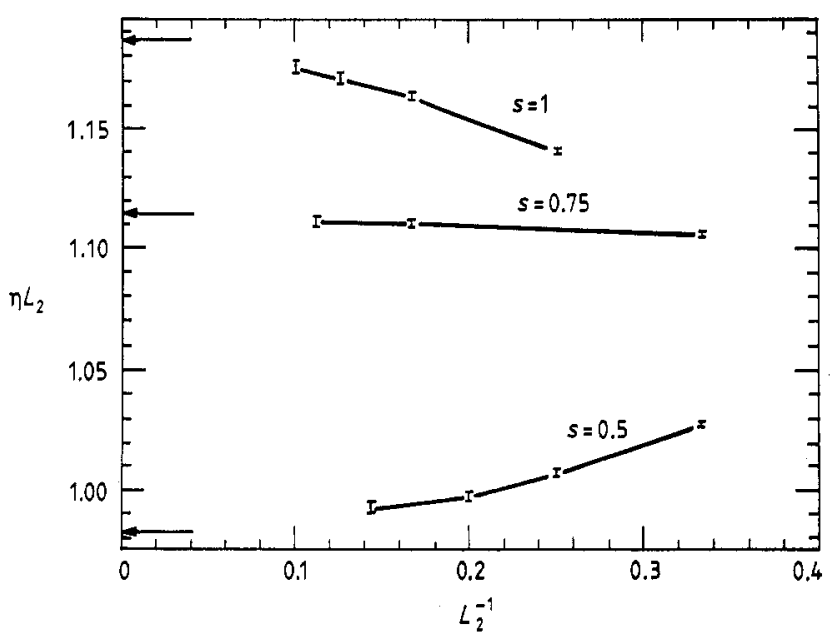

Figure 5. Universal amplitudes of the three-state Potts model. Arrows indicate the exact values of universal amplitudes at aspect ratios $s=1,0.75$ and 0.5 . The full line between the Monte Carlo data is a guide to the eye. 
energies $\eta\left(L_{2}\right)$. We obtained the values of $A(s)$ with an accuracy of $0.5 \%$ by extrapolation to $1 / L_{2}=0$, using (10). As expected, the effective correction-to-scaling exponent is between $y_{\mathrm{ir}, 1}=-\frac{4}{5}$ and $y_{\mathrm{ir}, 2}=-2.0$ (see above). The arrows along the vertical axis in figure 5 represent the exact values of $A(s)$ (Park and den Nijs 1988). The extrapolated numerical values are compared in table 1 with the exact values. Notice that the amplitudes of the corrections to scaling change sign as functions of the aspect ratio (the changes of slope in figure 5).

In figure 6, we plot the exact universal amplitude $A(s)$ as function of the aspect ratio $s$ of the Ising and three-state Potts model (Park and den Nijs 1988) together with our MC results.

Our results compare well with those of other methods. Monte Carlo renormalisation (MCRG) (Swendsen 1979) is one of the most accurate methods to determine critical exponents by means of MC simulations. Our results have a similar accuracy to those of MCRG (Swendsen 1982), especially the results obtained assuming knowledge of the exact value of the critical temperature (the MCRG results with which we compare used this information too). The algorithm that we used to calculate the interface free energies can certainly be improved in the future.

In this paper, we introduced a new Monte Carlo method of determining critical temperatures and universality classes of phase transitions. We intend to use this method

Table 1. Universal amplitudes of the three-state Potts model for several values of the aspect ratio $s$. The values in the second column are extrapolations to $L_{2}=\infty$ of the Monte Carlo data. The exact values in the third column are from Park and den Nijs (1988).

\begin{tabular}{lll}
\hline$s$ & Monte Carlo & Exact values \\
\hline 1 & $1.190 \pm 0.006$ & 1.18744 \\
0.75 & $1.113 \pm 0.005$ & 1.11384 \\
0.50 & $0.980 \pm 0.006$ & 0.98066 \\
\hline
\end{tabular}

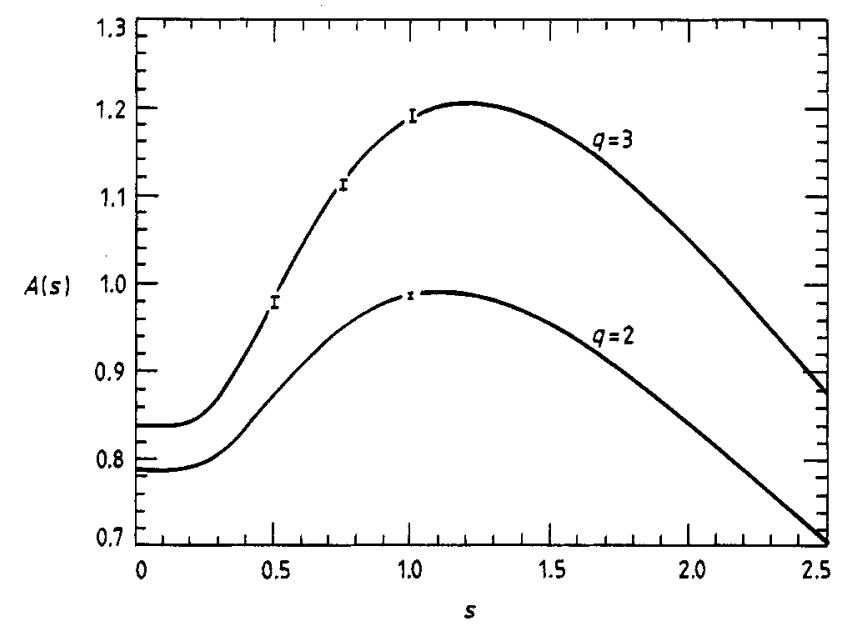

Figure 6. Universal finite-size scaling amplitudes for the Ising and three-state Potts model as function of the aspect ratio $s$. The full curves are the exact curves obtained by Park and den Nijs (1988). 
for systems with complex critical behaviour such as the helical and chiral Potts models (see Park et al 1986) and antiferromagnetic clock models (den Nijs 1985a). Here we tested and confirmed the feasibility of this method by applying it to simple models like the Ising and the three-state Potts model.

Next, we will test it on more complex systems but with still simple critical behaviours like the triangular Ising lattice-gas model and check whether the results still compare favourably with those of other numerical methods.

\section{Acknowledgments}

This research is supported by National Science Foundation Grant DMR 85-09392 and by the Alfred Sloan Foundation (MdN).

\section{References}

Barber M 1983 Phase Transitions and Critical Phenomena vol 8, ed C Domb and J L Lebowitz (London: Academic) ch 2

Belavin A A, Polyakov A M and Zamolodchikov A B 1984 J. Stat. Phys. 34763

Bennett C H 1976 J. Comput. Phys. 22245

Blöte H W J and den Nijs M 1988 Phys. Rev. B 371766

Cardy J L 1984 J. Phys. A: Math. Gen. 17 L385

___ 1987 Phase Transitions and Critical Phenomena vol 11, ed C Domb and J L Lebowitz (London: Academic) ch 2

den Nijs M 1979 J. Phys. A: Math. Gen. 121857

1983 Phys. Rev. B 271674

1984 J. Phys. A: Math. Gen. 17 L295

- 1985a Phys. Rev. B 324785

1985b J. Phys. A: Math. Gen. 18 L549

1988 Phase Transitions and Critical Phenomena vol 12, ed C Domb and J L Lebowitz (London: Academic) ch 2

den Nijs $M$ and Park $H$ unpublished

Ferdinand A E and Fisher M E 1969 Phys. Rev. 185832

Friedan D, Qiu Z and Shenker S 1984 Phys. Rev. Lett. 521575

Kaufman B 1949 Phys. Rev. 761232

Landau D P 1976 Phys. Rev. B 132997

Luck J M 1982 J. Phys. A: Math. Gen 15 L169

Mon K K and Jasnow D 1984 Phys. Rev. A 30670 1985 J. Stat. Phys. 41273

Mon K K and Nightingale M P 1985 Phys. Rev. B 316137

Nienhuis B 1987 Phase Transitions and Critical Phenomena vol 11, ed C Domb and J L Lebowitz (London: Academic) ch 1

Nightingale M P 1976 Physica 83A 561

1982 J. Appl. Phys. 537927

Nightingale M P and Blöte H W J 1983 J. Phys. A: Math. Gen. 16 L657

Park H and den Nijs M 1988 Phys. Rev. B 38565

Park H, Riedel E K and den Nijs M 1986 Ann. Phys., NY 172419

Privman V and Fisher M E 1984 Phys. Rev. B 30322

Swendsen R. H 1979 Phys. Rev. Lett. 42859

1982 Real-Space Renormalization ed T W Burkhardt and J M J van Leeuwen (Berlin: Springer) p 57 